\title{
Videogravação e videofeedback no ensino-aprendizagem de habilidades de exame físico
}

\author{
Video recording and videofeedback in teaching-learning of physical examination skills
}

\author{
Leonam Costa Oliveira' (1) | leonam.oliveira@iesvap.edu.br \\ Francisco Lucas Lima da Paz' (1) lucas.I.paz.ll@gmail.com \\ Ana Andreza Albuquerque Medeiros ${ }^{1}$ (D) andrezaaaam13@gmail.com \\ Eduardo Pinto Conceição² (1) edsmark@hotmail.com \\ Any Carolina Cardoso Guimarães ${ }^{1}$ (1) any.vasconcelos@iesvap.edu \\ Antonione Santos Bezerra Pinto' ${ }^{1}$ (D) antonione.pinto@iesvap.edu.br
}

\begin{abstract}
RESUMO
Introdução: O ensino de habilidades de exame físico, parte integrante e obrigatória dos currículos de escolas médicas, ocorre, tradicionalmente, com uma abordagem baseada na "demonstração e prática", e, embora existam outros modelos, não há, até o momento, nenhuma evidência de que um seja superior ao outro. Inovações nessa área são apontadas como caminho para suprir as deficiências de ensino-aprendizagem.

Objetivo: Este estudo teve como objetivos descrever a incorporação da videogravação e do videofeedback no ensino-aprendizagem de habilidades de exame físico e avaliar a eficácia desses recursos.

Método: Foi realizado um estudo historicamente controlado antes e depois da intervenção com alunos do primeiro ano de um curso de Medicina. O grupo de intervenção, em que se aplicaram a videogravação e o videofeedback, foi constituído de 91 alunos do semestre 2019.2, e o grupo controle contou com 72 alunos do semestre 2018.1. Ambas as turmas realizaram duas avaliações teóricas somativas (T1 e T2) e duas práticas, no formato de um exame clínico objetivo estruturado (OSCE). Na análise estatística comparativa das notas de ambas as turmas, utilizaram-se os testes não paramétricos da soma de postos de Wilcoxon-Mann-Whitney.

Resultado: A mediana das notas das avaliações práticas (primeiro e segundo OSCEs) de 2019 foram maiores do que as de 2018. Constatou-se ainda que a turma de 2019 teve uma evolução positiva das suas notas práticas passando de uma mediana de 11,6 no primeiro OSCE para 13,85 no segundo OSCE, o que também ocorreu com as provas teóricas $(p<0,05)$. Já na turma de 2018 , houve queda da mediana das notas da T1 para T2 e do primeiro OSCE para o segundo OSCE, mas sem significância estatística.

Conclusão: A incorporação da videogravação e videofeedback no ensino-aprendizagem de habilidades de exame físico entre graduandos do primeiro ano do curso de Medicina, em ambientes simulados, mostrou-se efetiva na melhora do desempenho dos discentes em avaliações teóricas e práticas. Essa abordagem se mostra ainda como meio de desenvolvimento e aplicação de uma aprendizagem motora observacional, reflexiva, experiencial e da metacognição no ensino-aprendizagem de habilidades de exame físico entre estudantes de Medicina.
\end{abstract}

Palavras-chave: Exame Físico; Habilidades; Gravação em Vídeo; Feedback; Educação Médica.

\begin{abstract}
Introduction: Physical examination skills, an integral and mandatory part of medical school curricula, are traditionally taught through an approach based on "demonstration and practice", and although other models exist, there is, to date, no evidence that one is superior to the other. Innovations in this area are pointed out as a way to supply deficiencies in teaching and learning.

Objectives: To describe the incorporation of video recording and video feedback in the teaching-learning of physical examination skills and to evaluate the effectiveness of such approach.

Method: A historically controlled study was carried out before and after the intervention with students in the first year of a medical course. The intervention group, in which video recording and video feedback was applied, consisted of 91 students from semester 2019.2 and the control group had 72 students from semester 2018.1. Both classes underwent two summative theoretical (T1 and T2) and two practical assessments, in the format of an objective structured clinical examination (OSCE). In the comparative statistical analysis of the grades of both classes, the non-parametric Wilcoxon and Mann Whitney rank test was used.
\end{abstract}

Results: The median of the marks of the practical evaluations (1st and 2nd OSCE) of the 2019 class were higher than those of 2018. It was also found that the class of 2019 showed a positive evolution in its practical marks, with the median score increasing from 11.6 in the 1st OSCE to 13.85 in the 2nd OSCE, which also happened with the theoretical tests $(p<0.05)$. In the class of 2018, there was a drop in the median mark from T1 to T2 and from the $1 s t$ OSCE to the 2nd OSCE, but without statistical significance.

Conclusion: The incorporation of video recording and video feedback in teaching-learning physical examination skills among first-year medical students, in simulated environments, proved to be effective in improving student performance in both theoretical and practical assessments. This approach is also shown as a means of developing and applying observational, reflective, experiential and metacognition motor learning in the teaching-learning of physical examination skills among medical students.

Keywords: Physical Examination; Skills; Video Recording; Feedback; Medical Education.

${ }^{1}$ Instituto de Educação Superior do Vale do Parnaíba, Parnaíba, Piauí, Brasil.

2 Universidade Federal do Delta do Parnaíba, Parnaíba, Piauí, Brasil.

Editora-chefe: Rosiane Viana Zuza Diniz. ～| Editor associado: Roberto Zonato Esteves.

Recebido em 03/01/22; Aceito em 16/01/22. | | Avaliado pelo processo de double blind review. 


\section{INTRODUÇÃO}

O ensino-aprendizagem de habilidades de exame físico faz parte de qualquer currículo de um curso de graduação em Medicina. Anteriormente introduzido nas escolas médicas brasileiras só após o ciclo básico e como ponto inicial do ciclo clínico, hoje, com a reformulação dos currículos a partir das Diretrizes Curriculares Nacionais (DCN) dos cursos de graduação em Medicina, os discentes do primeiro ano do curso médico já aprendem a realizar o exame de pacientes, pois trata-se de uma habilidade clínica básica, particularmente pertinente à prática clínica geral ${ }^{1-3}$.

O ensino de habilidades de exame físico ocorre, tradicionalmente, com uma abordagem baseada na "demonstração e prática"1,4. No entanto, existem outros modelos: modelo de quatro etapas de Peyton, modelo de seis etapas (adaptado de Peyton), modelo da execução silenciosa e modelo da descoberta colaborativa. Esses modelos geralmente apresentam em comum uma fase de demonstração, executada pelo professor, na qual se fornece ao aluno um conceito geral da habilidade a ser aprendida, o que está diretamente ligada à primeira fase do modelo psicológico de compreensão da aprendizagem de habilidades práticas, a fase dita cognitiva. Outro ponto semelhante é a decomposição de tarefas complexas em etapas sequenciais mais simples, relacionada intimamente à segunda fase do modelo psicológico, a de fixação prática das etapas mecânicas. $E$, por fim, tais modelos advogam a necessidade de um feedback construtivo do tutor ao aluno, para que assim este atinja a terceira fase desse modelo psicológico de aprendizagem, que é autonomia, na qual o aprendiz executa, por conta própria, a habilidade requerida. Alguns desses modelos também destacam a necessidade de vincular cada etapa/manobra do exame físico com a ciência básica e com a clínica, refletindo a teoria da aprendizagem experiencial de Kolb e fugindo da simples repetição mecânica de uma bateria de manobras ${ }^{5,6}$.

Não há, até o momento, nenhuma evidência de que um modelo seja superior ao outro, embora alguns deles melhorem pontos específicos da habilidade de exame físico, como o modelo de execução silenciosa que se mostrou mais eficaz no desempenho de tarefas motoras complexas e o modelo da descoberta colaborativa que melhorou o reconhecimento de achados do exame físico cardíaco. Uma revisão narrativa da literatura, depois de pesquisar e analisar os modelos propostos para o ensino das habilidades de exame físico, acabou por sugerir a necessidade do desenvolvimento de modelos práticos inovadores para esse proposito 5 .

A videogravação e o videofeedback surgiram como um suporte avaliativo às entrevistas médicas e como um recurso prático de autoavaliação quanto às habilidades de comunicação na relação médico-paciente. E, por conta disso, são consideradas ferramentas "padrão-ouro" de ensino-aprendizagem para o desenvolvimento das habilidades de entrevista clínica ${ }^{7}$. Pela possibilidade de sua utilização em outros contextos, essa pesquisa objetiva descrever a incorporação da videogravação e videofeeback no ensino-aprendizagem de habilidades de exame físico e avaliar sua eficácia.

\section{MÉTODOS}

O presente estudo baseou-se nas intervenções feitas durante as aulas práticas da disciplina Habilidades e Atitudes Médicas II (HAM II) do curso de Medicina no laboratório de simulação da Faculdade de Ciências Humanas, Exatas e da Saúde do Piauí (Fahesp), mantida pelo Instituto de Educação Superior do Vale do Parnaíba (lesvap), em que se utilizaram como ferramentas didáticas a videogravação e o videofeedback no ensino-aprendizagem das habilidades de exame físico.

A disciplina de HAM II, ministrada aos alunos do segundo semestre do curso de Medicina, aborda em sua ementa, entre outros objetivos, o ensino das técnicas de exame físico dos sistemas nervoso, osteomuscular, reprodutor, urinário e endócrino. Contempla ainda um treinamento sistemático, interativo e espiralar de habilidades técnicas, procedimentais e atitudes, buscando a realização com proficiência, pelos alunos, das técnicas do exame físico. No processo avaliativo da disciplina, são utilizadas avaliações formativas e somativas. A cada semestre, são aplicadas duas avaliações teóricas e duas práticas. As teóricas são somativas e consistem em uma prova com itens de múltipla escolha. Já as práticas são no formato de um exame clínico objetivo estruturado (objective structured clinical examination - OSCE), consideradas formativas, pois incluem um feedback individual, dado pelo avaliador, ao final de cada estação.

Nessa disciplina, os alunos, ao longo de 17 semanas, têm uma hora semanal dedicada a uma aula teórica, na qual participam todos os discentes matriculados, e a uma aula prática, ministrada a grupos de 12 a 15 estudantes no laboratório de simulação, onde são utilizados manequins e simuladores de baixa e média fidelidades. As aulas práticas são organizadas de forma que primeiro o professor demonstra o protocolo do exame físico e depois os alunos, aos pares e/ou usando simuladores, treinam a realização das manobras.

Participaram desta pesquisa 91 alunos do segundo semestre de 2019 que vivenciaram as aulas teóricas e práticas como descritas anteriormente, mas que tiveram a adição da videogravação e do videofeedback. Dessa forma, a didática executada nas aulas práticas da turma de 2019 foram estruturadas nas seguintes etapas (Figura 1): 
- Etapa 1: estudo teórico prévio: Estudo teórico prévio do assunto, utilizando livros-textos, protocolos e roteiros disponibilizados pelo professor com os passos de cada exame físico, além de uma aula teórica com todos os 91 alunos.

- Etapa 2: demonstração e prática: Aula prática, com grupos de 12 a 15 alunos, a respeito do conteúdo previamente estudado com demonstrações feitas pelo professor, seguidas da execução, pelos alunos, das técnicas de exame físico, sob supervisão docente. $\mathrm{Na}$ execução, os discentes realizavam as manobras em simuladores ou nos próprios pares. Pontua-se ainda que a abordagem pedagógica utilizada no ensino-aprendizagem do exame físico, entre os participantes deste estudo, foi a abrangente, ou seja, uma abordagem baseada em sistemas orgânicos, por se tratar de alunos do primeiro ano do curso médico em que o enfoque era a correlação da semiologia com a anatomia e fisiologia. Uma abordagem contextualizada em situações clínicas é outro caminho a ser considerado para favorecer o raciocínio clínico, podendo ser objeto de pesquisas futuras.

- Etapa 3: videogravação: Nessa etapa, os estudantes deveriam gravar vídeos com o conteúdo abordado nas aulas práticas, seguindo os parâmetros delimitados em sala de aula, como apresentação inicial, higienização correta das mãos, uso de equipamentos de proteção individual (EPI) e realização adequada das manobras, sempre respeitando a relação médico-paciente. Vale ressaltar que cada subgrupo de alunos era responsável por uma parte do exame físico de determinado sistema. Um exemplo prático é a avaliação do aparelho locomotor, em que um subgrupo produziu um vídeo com o exame da coluna cervical, enquanto o exame da coluna torácica, da lombar, do plexo braquial e lombossacral foi responsabilidade de outros subgrupos. Então, em um grupo de prática de 12 a 15 alunos, de quatro a cinco subgrupos eram formados para a confecção desses vídeos.

Além disso, também é importante destacar que, para a confecção dos vídeos, os alunos utilizavam seus próprios dispositivos móveis para gravação e usavam programas de edição gratuitos e de fácil manipulação, que não exigiam conhecimento avançado em tecnologia da informação. Eram disponibilizados horários livres no laboratório de simulação, onde os alunos podiam confeccionar os vídeos sob supervisão
Figura 1. As quatro etapas da metodologia aplicada no ensinoaprendizagem de habilidades de exame físico a alunos do primeiro ano de um curso de Medicina.

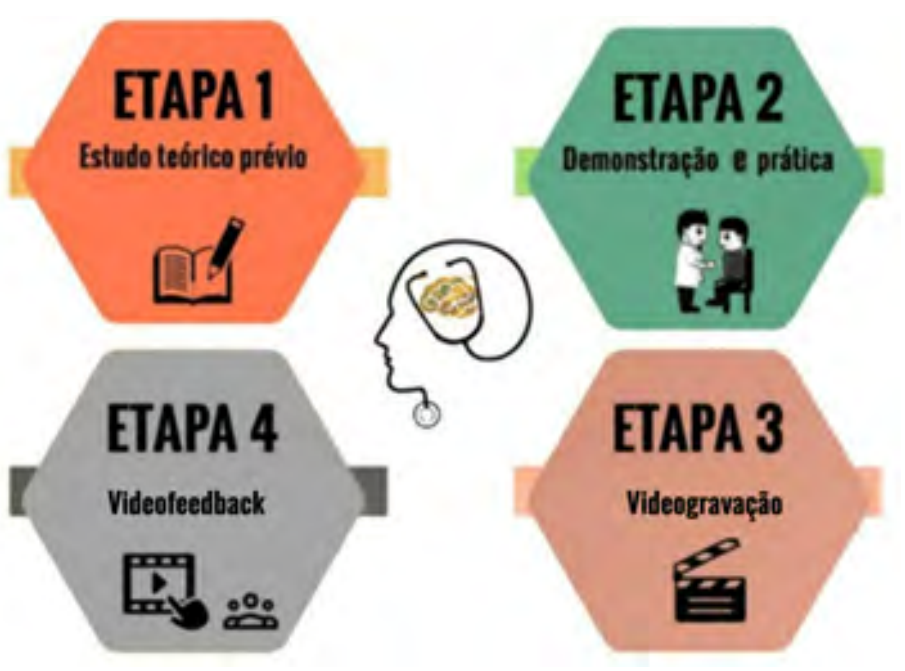

Fonte: Elaborada pelos autores.

dos monitores. Esses últimos eram orientados a apenas supervisionar e garantir a segurança, não podiam interferir na forma como os alunos estavam realizando as manobras. Era ainda determinado que os vídeos deveriam ser curtos, em média de três minutos, a depender da tarefa solicitada. Os alunos realizavam as manobras em seus pares e/ou em simuladores. O tempo para confecção dos vídeos eram em média de dez dias, pois deveriam ser apresentados ao final de uma outra aula prática. Então, o cronograma da disciplina era seguido. Por exemplo: findadas as aulas práticas do exame da coluna vertebral e de seus plexos, passava-se ao exame do sistema nervoso, e as apresentações dos vídeos do exame da coluna ocorriam quando a turma já estava em aulas práticas abordando outros sistemas.

- Etapa 4: videofeedback: Nessa etapa, realizava-se o feedback dos vídeos produzidos pelos alunos dos subgrupos. As gravações eram analisadas pelo professor em conjunto com o grupo de 12 a 15 pessoas, sendo apresentados cerca de dois a três vídeos ao final de cada aula prática. Estruturou-se o feedback da seguinte forma: após a apresentação de cada subgrupo, solicitavam-se aos próprios alunos produtores dos vídeos suas impressões sobre o trabalho que haviam executado, as suas fragilidades e seguranças. Em seguida, acontecia a avaliação interpares, em que os outros alunos deveriam apontar os acertos e as inadequações que identificaram em cada vídeo, podendo adquirir pontos com suas observações acerca do exame físico mostrado nos vídeos. E, por último, 
o professor relatava suas impressões acerca de cada vídeo apresentado pelos alunos, bem como sobre a avaliação feita pelos outros discentes que ficaram responsáveis pela execução de outros exames. Esse processo constituiu a técnica de videofeedback, na qual cada aluno foi capaz de rever seu comportamento durante a realização do exame físico, atentando-se aos erros cometidos durante esse procedimento e como e onde poderiam melhorar, além de também avaliarem a conduta do seu colega de turma.

Para avaliar a metodologia aplicada, compararam-se as notas das avaliações da turma de 2019 com as da turma de 2018. Dessa última, participaram 72 alunos que não tiveram a aplicação da videogravação e videofeedback, e, portanto, só passaram pelas etapas 1 e 2, já descritas, mas tiveram horários extras disponibilizados para treinamento nos laboratórios de simulação, sob supervisão de monitores Como se compararam dois grupos de alunos de diferentes semestres -em um dos grupos (turma de 2019), aplicou-se a intervenção; e, no outro (turma de 2018 - grupo controle, isso não ocorreu -, nossa pesquisa adotou o desenho de um estudo historicamente controlado antes e depois da intervenção, segundo a classificação de Boet et al. ${ }^{8}$.

Ambas as turmas realizaram duas avaliações teóricas e duas avaliações práticas. As avaliações teóricas consistiam em testes cognitivos contextualizados com itens de múltipla escolha e valiam de zero a dez. Já as avaliações práticas foram no formato de um OSCE e valiam de zero a 15 pontos. Cada aluno realizou seis estações durante o seu semestre vigente, sendo três estações na metade do período (primeiro OSCE) e outras três no final do período (segundo OSCE). No primeiro OSCE, as estações abordaram o exame físico da coluna lombar, do plexo lombossacral e do sistema nervoso. Já no segundo OSCE, foram cobrados os exames físicos da tireoide, do joelho e ginecológico. Os OSCEs eram estruturados com descrição da estação, checklist e pacientes padronizados. Os checklists consistiam em listas de verificação binária e continham de dez a 25 itens. Cada estação tinha duração de cinco minutos, e havia um minuto extra para feedback dado pelo avaliador da estação, caracterizando uma avaliação formativa. As estações eram quadruplicadas, havendo quatro corredores com as três estações de cada OSCE. Os avaliadores e pacientes padronizados receberam treinamento uma hora antes do início de cada OSCE, por meio de vídeo que continha o desempenho esperado e possíveis erros dos alunos. Também se repassavam os checklists e as falas e ações dos pacientes padronizados. Os avaliadores eram professores de outras disciplinas da faculdade, e o professor do módulo de HAM
Il e os autores desta pesquisa não foram avaliadores nesses OSCEs, atuando apenas na organização, no treinamento, na logística e na supervisão da aplicação dessa prova. Os avaliadores ficavam presentes durante toda a passagem dos discentes pela estação, realizando uma observação direta, o que permitiu avaliar os domínios de aprendizagem dos alunos e realizar feedback.

Com relação à validade de conteúdo, ou seja, o grau em que o conteúdo dos instrumentos avaliativos refletia adequadamente o construto que estava sendo medido, esclarece-se que tais instrumentos são construídos pelo professor da disciplina de HAM II e depois enviados para avaliação e revisão de duas comissões do lesvap. As provas teóricas são revisadas pela comissão de revisão de provas (Corp), composta por outros cinco professores que examinam todos os itens e avaliam se as questões construídas apresentam enunciado, comando e alternativas, se são itens contextualizados e não factuais, e se contemplam os temas da ementa da disciplina. Já as estações e os checklists do OSCE são examinados e revisados por professores do Núcleo de Apoio Pedagógico e Estruturante Docente (Naped) que avaliam a proporção dos conteúdos abordados no OSCE em relação aos ministrados no semestre, a qualidade das estações e as listas de verificações. A consistência interna dos OSCEs foi avaliada pelo alfa de Cronbach. Para esta análise, valores menores que 0,21 são considerados de consistência interna pequena; de 0,40 a 0,21, razoável; de 0,60 a 0,41, moderada; e maior que 0,61, substancial. Já em relação às avaliações teóricas, efetuou-se o cálculo do índice de discriminação - ID (ponto bisserial) dos itens como forma de se realizar uma análise psicométrica dos resultados dessas provas. Valores maiores ou igual a 0,40 são classificados com ID muito bom; de 0,3 a 0,39, bom; de 0,20 a 0,29, médio; e inferior ou igual a 0,19, fraco 9 .

As notas obtidas pelos alunos de 2018 e 2019 na primeira e segunda avaliações teóricas (T1 e T2) e no primeiro e segundo OSCEs foram digitadas em arquivos específicos, e realizou-se a análise estatística por meio do programa R 3.5.2 - The R-project for statistical computing. Para avaliar a normalidade, aplicou-se o teste de Shapiro-Wilk. O conjunto das notas das avaliações T1 e T2, do primeiro OSCE de 2018, de T1 e T2, e do segundo OSCE de 2019 mostrou níveis de significância da estatística W menores que os valores críticos (valor $p$ inferior a 0,05 ), ou seja, esses valores não seguiam fidedignamente uma distribuição normal. Já as avaliações do segundo OSCE de 2018 e do primeiro OSCE de 2019 mostraram dados com distribuição normal. Como alguns dados apresentaram distribuição normal e outros não, optou-se por aplicar testes não paramétricos como método estatístico.

Para a comparação das notas das turmas de 2018 com as de 2019, assim como para a comparação das notas das primeiras e segundas avaliações teóricas e práticas de cada 
uma dessas turmas, aplicou-se o teste da soma de postos de Wilcoxon-Mann-Whitney. As amostras das notas, de cada uma das avaliações (T1, T2, primeiro OSCE e segundo OSCE) e de cada uma das turmas, foram agrupadas em um mesmo conjunto, o qual foi submetido a um processo de ordenação e ranqueamento, obtendo-se assim uma paridade entre o dado originalmente observado e seu respectivo posto. A ordenação das observações foi feita da menor para a maior, sendo atribuído um posto a cada uma. Em seguida, realizou-se a soma dos postos correspondentes a cada um dos grupos. Terminado esse processo, o conjunto maior de dados foi novamente revertido aos grupos iniciais, agora contando, cada grupo, com uma nova variável, representada pelo posto ocupado pelo dado original e que foi utilizada para computação dos resultados. Procedeu-se então ao cálculo da estatística dos testes W e U e do $p$-valor.

Esta pesquisa utilizou apenas um banco de dados e não realizou entrevistas. Os dados utilizados foram solicitados à Fahesp que forneceu anuência e autorização para a utilização do seu banco de notas. Tais dados foram utilizados em sua totalidade, não havendo a identificação de nenhum aluno. Por esses motivos, não se solicitou o parecer do Comitê de Ética em Pesquisa.

\section{RESULTADOS}

A mediana das notas das avaliações práticas (primeiro e segundo OSCEs) e da T2 de 2019 foi maior que a mediana de 2018. Quando se analisaram as provas teóricas, a mediana das notas da T1 de 2019 foi menor que a mediana de 2018 (Tabela 1). Com relação à evolução das notas, dentro da mesma turma, ao longo do semestre, constatou-se que a turma de 2019 teve uma evolução positiva das suas notas práticas, passando de uma mediana de 11,6 no primeiro OSCE para 13,85 no segundo OSCE, o que também ocorreu com as provas teóricas, tudo isso com significância estatística. Já na turma de 2018, houve queda da mediana das notas da T1 para T2 e do primeiro OSCE para o segundo OSCE, mas sem significância estatística (Tabela 1).

Tabela 1. Mediana das notas obtidas pelos alunos dos semestres de 2018 e 2019 na disciplina de Habilidades e Atitudes Médicas Il e a comparação entre as primeiras e segundas avaliações teóricas e práticas de cada semestre, utilizando o teste de Wilcoxon-Mann-Whitney.

\begin{tabular}{|c|c|c|c|c|c|c|}
\hline & & Mediana & Valor mínimo & Valor máximo & Estatística W & $p$ \\
\hline \multirow{4}{*}{$2018.1(n=72)$} & Teórica 1 & 7,55 & 4 & 10 & \multirow{2}{*}{1211.5} & \multirow{2}{*}{0,917} \\
\hline & Teórica 2 & 7 & 3 & 9 & & \\
\hline & $1^{\circ} \mathrm{OSCE}$ & 10,65 & 5,2 & 12,7 & \multirow{2}{*}{1270.5} & \multirow{2}{*}{0,446} \\
\hline & $2^{\circ} \mathrm{OSCE}$ & 10,5 & 5,35 & 13,6 & & \\
\hline \multirow{4}{*}{$2019.2(n=91)$} & Teórica 1 & 7,2 & 2,3 & 9,3 & \multirow{2}{*}{200.5} & \multirow{2}{*}{$<0,05$} \\
\hline & Teórica 2 & 9 & 4,25 & 10 & & \\
\hline & $1^{\circ} \mathrm{OSCE}$ & 11,6 & 7,15 & 15 & \multirow{2}{*}{85.5} & \multirow{2}{*}{$<0,05$} \\
\hline & $2^{\circ} \mathrm{OSCE}$ & 13,85 & 10,6 & 14,85 & & \\
\hline
\end{tabular}

OSCE - exame clínico objetivo estruturado.

Fonte: Elaborada pelos autores.

Tabela 2. Comparação entre os escores medianos dos postos das notas das avaliações T1, T2, primeiro OSCE e segundo OSCE entre os grupos 2018 e 2019 utilizando o teste de Mann-Whitney.

\begin{tabular}{|c|c|c|c|c|c|}
\hline Grupo & Avaliação & Mediana & Estatística U & Escore Z & $p$ \\
\hline 2018 & \multirow{2}{*}{$1^{\circ} \mathrm{OSCE}$} & 10,65 & \multirow{2}{*}{2135} & \multirow{2}{*}{-3.812} & \multirow{2}{*}{$<0,05$} \\
\hline 2019 & & 11,6 & & & \\
\hline 2018 & \multirow{2}{*}{$2^{\circ} \mathrm{OSCE}$} & 10,5 & \multirow{2}{*}{291.5} & \multirow{2}{*}{-9.973} & \multirow{2}{*}{$<0,05$} \\
\hline 2019 & & 13,85 & & & \\
\hline 2018 & \multirow{2}{*}{ Teórica 1} & 7,55 & \multirow{2}{*}{3937.5} & \multirow{2}{*}{2.223} & \multirow{2}{*}{$<0,05$} \\
\hline 2019 & & 7,2 & & & \\
\hline 2018 & \multirow{2}{*}{ Teórica 2} & 7 & \multirow{2}{*}{1138} & \multirow{2}{*}{-7.304} & \multirow{2}{*}{$<0,05$} \\
\hline 2019 & & 9 & & & \\
\hline
\end{tabular}

OSCE - exame clínico objetivo estruturado.

Fonte: Elaborada pelos autores. 
Já o somatório dos postos do primeiro e segundo OSCES, ranqueados a partir das notas, foi maior no grupo de alunos de 2019 do que no grupo de 2018, com diferença estatística entre os grupos (Tabela 2).

As consistências internas das estações do OSCE no ano de 2018 foram 0,732, 0,622, 0,865, 0,825, 0,299 e 0,634. Já no ano de 2019, as confiabilidades foram 0,445, 0,446, $0,673,0,380,0,878$ e 0,705. Portanto, todas apresentaram confiabilidade aceitável (acima de 0,21), e oito das 12 estações tiveram confiabilidade substancial (acima de 0,61) ${ }^{9}$. Já na análise das provas teóricas do ano de 2018, 25\% dos itens tiveram um ID classificado como muito bom; $15 \%$, como bom; $25 \%$, como médio; e 35\%, como fraco. Nas provas teóricas de 2019, 30\% dos itens tiveram ID muito bom; 20\%, bom; 15\%, médio; e $35 \%$, fraco.

\section{DISCUSSÃO}

A metodologia de quatro etapas, adotada no ensinoaprendizagem de habilidades de exame físico nesta pesquisa, que incorpora a videogravação e o videofeedback, mostrouse eficaz em melhorar o desempenho de alunos do primeiro ano do curso de Medicina nas avaliações práticas (OSCE). Eles apresentaram ainda uma melhora significativa de suas notas, da primeira para a segunda avaliação dentro do mesmo semestre, o que não foi observado entre os alunos que não vivenciaram tal metodologia. Isso demonstra que a possibilidade de os discentes confeccionarem vídeos e receberem feedback, visualizando o que executam, pode contribuir para que melhorem sua metacognição, ou seja, que consigam perceber suas qualidades e fragilidades e possam agir sobre elas, melhorando seu desempenho.

$\mathrm{Na}$ análise das notas das avaliações teóricas, percebese que, apesar de a mediana das notas da T1 dos alunos de 2019.2 ser mais baixa do que da turma de 2018.1, eles tiveram uma melhora mais significativa do seu desempenho dentro do mesmo semestre e obtiveram uma mediana de notas na T2 significativamente superior. Isso mostra que a metodologia adotada de videogravação e videofeedback pode também contribuir para a cognição e não só para habilidades técnicas e mecânicas. No entanto, uma análise mais aprofundada faz-se necessária, pois tal metodologia foi desenhada principalmente para as atividades práticas, simuladas, em laboratório.

A videogravação e o videofeedback são metodologias já muito utilizadas no ensino de habilidades de comunicação, mas ainda pouco exploradas no ensino do exame físico. Spence et al. ${ }^{10}$ aplicaram-nas no treinamento de ressuscitação cardiopulmonar, em que compararam duas coortes de alunos, em uma delas foi aplicado o videofeedback. Esse estudo mostrou uma eficácia significativamente maior do feedback feito por meio de vídeo quando comparado ao feedback verbal, o que levou a um aprimoramento das habilidades individuais ${ }^{10}$. Um outro ensaio clínico randomizado, que teve por finalidade analisar a eficácia do feedback por vídeo em comparação com o feedback tradicional na assistência ao suporte básico de vida, evidenciou notória evolução do grupo que sofreu a intervenção em relação ao grupo controle ${ }^{11}$. Isso também foi constatado em uma pesquisa com acadêmicos de odontologia que, por meio do sistema avaliativo do OSCE, observou melhora no desempenho de habilidades práticas dos estudantes que eram submetidos ao videofeedback ${ }^{12}$. Dessa forma, esses autores apontam para a possibilidade do seu uso no ensino de habilidades psicomotoras, o que foi objeto de estudo da nossa pesquisa, a qual encontrou resultados favoráveis, e com significância estatística, para a uso da videogravação e videofeedback no ensino de habilidades de exame físico.

As quatro etapas desenvolvidas nessa metodologia foram baseadas em teorias de aprendizagem utilizadas em educação médica. Na primeira e segunda etapas, há o estudo teórico e a demonstração e prática de técnicas/manobras do exame físico sob supervisão e orientação do professor. Respeitam-se assim as três fases da compreensão psicológica da aprendizagem de habilidades: a cognitiva, a fixação prática das etapas mecânicas e a autonomia. Ressalta-se que essas duas primeiras etapas ocorrem de forma integrada com outros componentes curriculares, como anatomia e fisiologia, e têm uma abordagem baseada em sistemas orgânicos na qual uma bateria de manobras é ensinada, caracterizando assim uma abordagem pedagógica abrangente no ensino de habilidades de exame físico ${ }^{4}$.

Na segunda etapa dessa metodologia, o aluno aprende observando o professor, mostrando assim um predomínio da aprendizagem dita observacional e mais precisamente da aprendizagem motora observacional e da aprendizagem de sequência motora, em que há observação de uma ação, as técnicas/manobras de exame físico, visando ao refinamento do desempenho motor. Segundo a neurociência cognitiva, isso está intimamente ligado ao sistema de neurônios-espelhos, recompensa e memória, que, por sua vez, está envolvido na percepção da ação e desempenho, e, portanto, na experiência e no aprendizado ${ }^{13}$.

Ao ser incorporada a videogravação (terceira etapa) a essa metodologia descrita, oportuniza-se ao discente vivenciar novamente cada uma das fases da compreensão psicológica da aprendizagem, mas com o diferencial de ser em um novo formato, em uma nova experiência, em que o aluno executa a cognição, a demonstração e a prática com seus pares, suscitando interações e trabalho em equipe. Tudo isso com um maior grau de autonomia, uma vez que, no momento da produção dos vídeos, o professor não está presente. 
A aprendizagem experiencial é um processo em que a aprendizagem ocorre por meio de uma experiência ${ }^{14}$. Nesse sentido, com essa terceira etapa uma nova experiência de aprendizagem é acrescida ao processo educacional do aluno, que, além do encontro no laboratório de simulação, tem agora esse segundo momento, com esse novo formato, o de videogravação, em que, além de executar e repetir manobras, deve refletir sobre elas para poder chegar a uma execução desejável, uma vez que a experiência por si só não é suficiente para que a aprendizagem ocorra. Em um estudo com graduandos de Medicina, na disciplina de Pediatria, em que foi utilizado o feedback de gravação de vídeo de uma consulta, com coleta de anamnese e execução do exame físico, 73\% dos discentes acreditavam que a sua auto-observação, por meio do vídeo, influenciou o desenvolvimento de suas habilidades clínicas ${ }^{15}$.

O uso do conceito de reflexão na educação médica tem se tornado recorrente e consagrado, sendo uma abordagem indicada por várias diretrizes nacionais e internacionais de cursos de graduação em Medicina. No entanto, a abordagem a ser utilizada para sua efetiva aplicação cotidiana ainda é motivo de discussão ${ }^{14}$. Na quarta etapa (videofeedback), quando o grupo que confecciona o vídeo apresenta à turma seu trabalho e eles próprios e os outros alunos apontam os pontos fortes e as fragilidades das técnicas/manobras apresentadas, é possível promover, nesse momento de aprendizagem, a reflexão individual e a coletiva, e também trabalhar a metacognição, pois isso estimula nos alunos a capacidade deles de prever o próprio desempenho nas tarefas. Nesse mesmo sentido, estudos que abordaram o videofeedback no ensino-aprendizagem de habilidades de comunicação apontaram que esse é um caminho para uma aprendizagem experiencial e autoavaliação reflexiva ${ }^{16}$. Um outro estudo, que abordou sua aplicação no próprio exame físico, evidenciou que $80 \%$ dos discentes concordaram que o feedback por vídeo, de professores e de seus pares, melhorou suas habilidades clínicas ${ }^{15}$. Teoriza-se assim que a reflexão e a metacognição monitoram e avaliam o processo cognitivo, o que é essencial para uma aprendizagem mais ampla, superando a simples identificação e aquisição de novas habilidades ${ }^{14}$. Nesse sentido, a videogravação e o videofeedback são os meios adotados, por essa metodologia de ensino-aprendizagem de habilidade de exame físico, para o desenvolvimento da reflexão.

As etapas três e quatro permitem também um movimento cíclico no ensino-aprendizagem das habilidades de exame físico, uma vez que os alunos, tendo cumprido as etapas um e dois, ou seja, já tendo estudado, demonstrado e praticado técnicas/manobras do exame físico, têm que novamente executá-las durante a produção dos vídeos e em seguida revê- las no videofeedback, criando assim diferentes oportunidades, no mesmo semestre, para o discente experienciar, repetir, memorizar, refletir, abstrair e apreender habilidades de exame físico. A "reobservação" é considerada uma boa prática para o feedback e a aprendizagem ${ }^{17}$. Esse desenho tem influências do ciclo de aprendizagem experiencial de Kolb e de suas quatro fases: experiência concreta, reflexão, conceitualização abstrata e nova experiência ativa ${ }^{6,14}$, bem como no conceito de feedback como um ciclo em que há entrega de uma informação, uma reação e uma consequência para melhoria das respostas ${ }^{17}$.

Esta pesquisa descreveu e avaliou uma proposta de metodologia de ensino-aprendizagem de habilidades de exame físico, dividida em quatro etapas, aplicada aos alunos do primeiro ano do curso de Medicina que vivenciam um ambiente simulado de aprendizagem, preparando-os para a realização futura do exame físico em pacientes. A importância de se valer de pacientes reais à beira do leito para o ensino do exame físico é enfatizada por diversos autores, no entanto utilizá-los com alunos dos primeiros anos do curso, iniciantes na aprendizagem de tal habilidade, pode ser desafiador, complexo e até trazer prejuízos à aprendizagem, pois podem ocorrer situações como o paciente estar indisposto, estar em programação para a realização de algum exame complementar, não aceitar a repetição das manobras por vários alunos ou ainda tratar-se de caso de complexidade, do ponto de vista médico, não adequado ao momento curricular no qual os discentes se encontram ${ }^{4,18,19}$. Por meio da simulação e de simuladores, é possível contornar alguns desses problemas, uma vez que esses recursos podem reproduzir sinais patológicos específicos, permitir a prática e repetição de habilidades psicomotoras e cognitivas, e até gerar dados de desempenho individual, além da possibilidade de eles serem utilizados antes de pacientes reais e com alunos do início do curso ${ }^{4,19}$.

Como limitação do nosso estudo, destaca-se a falta de dados do desempenho dos alunos em longo prazo e em pacientes reais, uma vez que só foram avaliados no semestre em que cursaram a disciplina de HAM II. Apesar de o OSCE ser um instrumento que prediz o desempenho dos alunos em situações reais, trabalhos que efetivamente abordem esse aspecto podem elucidar tais indagações. Outro desafio encontrado na experiência usando a videogravação e o videofeedback foi a inibição de alguns alunos ao serem gravados e terem seu vídeo exposto em sala de aula. Ademais, outra limitação existente foi a pouca disponibilidade de horários extracurriculares pela instituição de ensino superior para utilização do laboratório de simulação realística, pois o local também era utilizado para realização de atividades curriculares, o que dificultava a reserva do local para a gravação dos vídeos e para uso dos equipamentos necessários. 


\section{CONCLUSÕES}

A incorporação da videogravação e do videofeedback no ensino-aprendizagem de habilidades de exame físico entre graduandos do primeiro ano do curso de Medicina, em ambientes simulados, mostrou-se efetiva na melhora do desempenho dos alunos em avaliações teóricas e práticas. Essa abordagem é um meio de desenvolvimento e aplicação de teorias de aprendizagem, como a aprendizagem reflexiva, experiencial e a metacognição, nas disciplinas/módulos de habilidades de exame físico das escolas médicas.

A metodologia de quatro etapas para o ensinoaprendizagem de habilidades de exame físico, aqui relatada, alia o estudo cognitivo, a demonstração e a prática (primeira e segunda etapas) a uma aprendizagem reflexiva e metacognitiva (terceira e quarta etapas), em que os alunos visualizam e discutem o próprio desempenho e o de seus pares. Como as etapas 3 e 4 permitem a repetição da performance do aluno na execução das manobras, ou seja, da cognição, demonstração e prática, traz-se também uma aprendizagem cíclica para dentro de um mesmo componente curricular, além de serem etapas que oportunizam diferentes experiências de aprendizagem, tanto em laboratório, com o professor, como durante a confecção dos vídeos, com os pares.

\section{CONTRIBUIÇÃO DOS AUTORES}

Leonam Costa Oliveira contribuiu na elaboração do projeto, na revisão da literatura, na coleta e análise dos dados, e na confecção do manuscrito. Francisco Lucas Lima da Paz, Ana Andreza Albuquerque Medeiros, Any Carolina Cardoso Guimarães e Antonione Santos Bezerra Pinto contribuíram na revisão da literatura, na coleta dos dados e na confecção do manuscrito. Eduardo Pinto Conceição contribuiu na elaboração do projeto, na análise dos dados e na revisão do manuscrito.

\section{CONFLITO DE INTERESSES}

Declaramos não haver conflito de interesses.

\section{FINANCIAMENTO}

Programa de iniciação científica da FAHESP/IESVAP.

\section{REFERÊNCIAS}

1. Dull $P$, Haines DJ. Methods for teaching physical examination skills to medical students. Fam Med. 2003;35(5):343-8.

2. Kahwage Neto SG, Braga TKK, Portella MB, Andriolo RB. O ensino de habilidades clínicas e a aplicabilidade de um guia simplificado de exame físico na graduação de Medicina. Rev Bras Educ Med. 2017;41(2):299-309.

3. Costa GPO, França KAND, Santos MAL, Guilherme JG, Medeiros JGMD, Silva Júnior EAD. Dificuldades iniciais no aprendizado do exame físico na percepção do estudante. Rev Bras Educ Med. 2020;44(1):e027.

4. Uchida T, Park YS, Ovitsh RK, Hojsak J, Gowda D, Farnan JM, et al. Approaches to teaching the physical exam to preclerkship medical students: results of a national survey. Acad Med. 2019;94(1):129-34.

5. Easton G, Stratford-Martin J, Atherton H. An appraisal of the literature on teaching physical examination skills. Educ Prim Care. 2012;23(4):246-54.

6. Yardley S, Teunissen PW, Dornan T. Experiential learning: AMEE Guide No. 63. Med Teach. 2012; 34(2):e102-15.

7. Noble LM, Scott-Smith W, O'Neill B, Salisbury H. Consensus statement on an updated core communication curriculum for UK undergraduate medical education. Patient Educ Couns. 2018;101(9):1712-9.

8. Boet S, Sharma S, Goldman J, Reeves S. Review article: medical education research: an overview of methods. Can J Anaesth. 2012;59(2):159-70.

9. Landis JR, Koch GC. The measurement of observer agreement for categorical data. Biometrics.1977;33(1):159-174.

10. Spence AD, Derbyshire S, Walsh IK, Murray JM. Does video feedback analysis improve CPR performance in phase 5 medical students? BMC Med Educ. 2016;16(1):203-210.

11. Sopka S, Hahn F, Vogt L, Pears KH, Rossaint R, Rudolph J, et al. Peer video feedback builds basic life support skills: a randomized controlled noninferiority trial. Plos One. 2021;16(7):e0254923.

12. Seifert LB, Herrera-Vizcaino C, Herguth P, Sterz J, Sader R. Comparison of different feedback modalities for the training of procedural skills in oral and maxillofacial surgery: a blinded, randomized and controlled study. BMC Med Educ. 2020;20(1):330-340.

13. Ramsey R, Kaplan DM, CrossES. Watch and learn:the cognitive neuroscience of learning from others' actions. Trends Neurosci. 2021;44(6):478-491.

14. Sandars J. The use of reflection in medical education: AMEE Guide No. 44 Med Teach. 2009;31(8):685-95.

15. Paul S, Dawson KP, Lanphear JH, Cheema MY. Video recording feedback a feasible and effective approach to teaching history-taking and physical examination skills in undergraduate paediatric medicine. Med Educ. 1998;32(3):332-6.

16. Dohms MC, Collares CF, Tibério IC. Video-based feedback using real consultations for a formative assessment in communication skills. BMC Med Educ. 2020;20(1):57-66.

17. van de Ridder JM, Stokking KM, McGaghie WC, ten Cate OT. What is feedback in clinical education? Med Educ. 2008;42(2):189-97.

18. Elder A, Chi J, Ozdalga E, Kugler J, Verghese A. A piece of my mind. The road back to the bedside. JAMA. 2013;310(8):799-800.

19. Uchida T, Achike FI, Blood AD, Boyle M, Farnan JM, Gowda D, et al. resources used to teach the physical exam to preclerkship medical students: results of a national survey. Acad Med. 2018;93(5):736-41. 